\title{
Associations Between Glycemic Control, Depressed Mood, Clinical Depression, and Diabetes Distress Before and After Insulin Initiation: An Exploratory, Post Hoc Analysis
}

\author{
Haya Ascher-Svanum · Anthony Zagar · Dingfeng Jiang • \\ Dara Schuster · Henry Schmitt · Ellen B. Dennehy • \\ David M. Kendall · Joel Raskin · Robert J. Heine \\ To view enhanced content go to www.diabetestherapy-open.com \\ Received: March 6, 2015 / Published online: July 10, 2015 \\ (C) The Author(s) 2015. This article is published with open access at Springerlink.com
}

\section{ABSTRACT}

Introduction: Although depression is often associated with poor glycemic control in patients with type 2 diabetes mellitus (T2DM), this observation has been inconsistent. This exploratory, post hoc analysis investigated associations between depression parameters and glycemic control using data from a 24-month, prospective, observational, noninterventional study evaluating glycemic response following insulin initiation for T2DM.

Electronic supplementary material The online version of this article (doi:10.1007/s13300-015-0118-y) contains supplementary material, which is available to authorized users.

H. Ascher-Svanum $(\bowtie) \cdot$ A. Zagar · D. Jiang · D. Schuster - E. B. Dennehy · D. M. Kendall . J. Raskin

Eli Lilly and Company, Indianapolis, IN, USA e-mail: haya@lilly.com

H. Schmitt

Eli Lilly Benelux, Brussels, Belgium

E. B. Dennehy

Department of Psychological Sciences, Purdue

University, West Lafayette, IN, USA

R. J. Heine

Eli Lilly Canada, Toronto, ON, Canada
Methods: We analyzed data from a 24-month, prospective, observational study that evaluated glycemic response in patients with T2DM who initiated insulin therapy $(N=985)$ in 5 European countries. Secondary measures included patient-reported diagnosis of depression at baseline, severity of depressed/ anxious mood (EuroQol (EQ)-5D item) and diabetes-related distress (Psychological Distress domain of the Diabetes Health Profile, DHP-18). The latter two measures were assessed at baseline and 5 time points throughout the study. Glycemic control was measured by glycated hemoglobin (HbA1c) at these same time points. Analyses employed $t$ tests to assess the unadjusted baseline difference in HbA1c between patients with and without the respective depression parameter. The potential effect of demographic and clinical confounding variables was controlled through a linear model structure. Patient HbA1c levels were analyzed by presence/absence of a history of diagnosed depression, depressed mood, and diabetesrelated distress.

Results: Patients with higher depression parameters or distress at baseline had significantly higher rates of microvascular 
complications at baseline. Patients with a history of diagnosed depression or high diabetes-related distress had higher HbA1c than patients without. HbA1c of patients with or without depressed mood was not significantly different at baseline. The proportion of patients with depressed mood declined after insulin initiation, whereas the proportion of patients with high diabetesrelated distress did not significantly change. HbA1c improved following insulin initiation, regardless of presence/absence of studied depression/distress parameters at baseline.

Conclusion: History of diagnosed depression, diabetes-related distress, and depressed mood were associated with a higher rate of microvascular complications. Diagnosed depression and diabetes-related distress also showed higher HbA1c at baseline when insulin was initiated. Insulin therapy improved glycemic control, while preexisting depressed mood declined and diabetes-related distress remained unchanged.

Keywords: Depressed mood; Depression; Diabetes distress; Glycemic control; Insulin therapy

\section{INTRODUCTION}

Depression affects approximately 20-25\% of patients with diabetes [1], with rates of major depressive disorder estimated at $12 \%$ and depressive symptoms at $15-35 \%$ [2]. The presence of depressive symptoms is associated with a poorer quality of life in patients with diabetes and has been shown to be associated with poorer glycemic control and diabetes complications [3-7]. Similar associations have been reported with subthreshold depression [7].

The relationship between glycemic control and depression is likely bidirectional, but the causal pathways remain incompletely understood [8]. Although poorer self-care among diabetes patients (e.g., adherence to lifestyle recommendations and glucose monitoring) is regarded as a potential contributor to poor glycemic control over time, it cannot fully account for poor control, as depression may also impact stress pathways which in turn can affect glycated hemoglobin (HbA1c) levels [9, 10]. However, interventions aimed at reducing depression among patients with diabetes have not led to the reduction in HbA1c or improvements in self-care behaviors, and conversely, an intervention trial to improve diabetes self-care and glycemic control in elderly patients with diabetes did not lead to reduction in depression $[11,12]$.

Although major depressive disorder and depressive symptoms have generally been considered to be associated with poor glycemic control in type 2 diabetes mellitus (T2DM), the available data from cross-sectional studies on this association are inconsistent and methodological approaches vary across studies $[2,9,13-25]$.

Diabetes-related distress refers to the emotional burden that may be an aspect of managing a chronic illness, and can be found in both those with diabetes and their caregivers [26]. It is different from the clinical experience of depression [27-30], as it may manifest more in emotional reactions to diabetes and its treatment. High levels of distress have been significantly linked to elevated HbA1c [29, 31]. Importantly, a prospective, observational, noninterventional study by Fisher and colleagues [8] concluded that diabetes distress, but not clinical depression or depressive symptoms, is associated with poorer glycemic control in both cross-sectional and longitudinal analyses. That 18-month study included a relatively homogeneous group of patients with T2DM 
who participated in diabetes education programs and had a reasonable level of glycemic control (average HbA1c of $7.2 \%$ ). It is important to replicate these findings in a more heterogeneous group of patients to fully understand the relationships between affective parameters and glycemic control in patients with T2DM. It is also important to study the association between $\mathrm{HbA} 1 \mathrm{c}$ and depression or distress parameters prior to and following patients' transition to insulin therapy. Barriers to timely intensification of treatment to insulin therapy in patients with poor glycemic control also encompass patients' psychological insulin resistance [32, 33], which contributes to long delays in the start of insulin therapy [34-37]. Psychological resistance to insulin is a multifaceted, encompassing fear of insulin and the potential side effects of hypoglycemia and weight gain, complexity of insulin treatment and regimens, need to self-monitor blood glucose to adapt doses, and emotional factors such as anxiety or depression symptoms [32, 33, 38-41].

Inconsistencies in our understanding regarding the link between glycemic control and depression may be due, in part, to the reliance on diverse measures of depression. These include a previous depression diagnosis (either from claims data, medical records, or self-reported), results from depression diagnostic screening tools (e.g., the Center for Epidemiologic Studies-Depression scale, the Patient Health Questionnaire) or full batteries, e.g., the Structured Clinical Interview for DSM-IV [Diagnostic and Statistical Manual of Mental Disorders, 4th Edition] Diagnosis, scores from clinical assessments of depression symptoms (e.g., the Quick Inventory of Depressive Symptomatology, the MontgomeryAsberg Depression Rating Scale) or measures of diabetes-related distress [27, 42]. A report from the National Institute of Diabetes, Digestive and
Kidney Diseases on diabetes and depression points out that the definition of depression varies across studies due to the variability of measurement and use of undefined terminology [43].

To help clarify the link between glycemic control and depression, this study investigated the association between three types of depression/diabetes distress parameters (depression diagnosis, depressed mood, and diabetes-related distress) over 24 months in patients with T2DM initiating insulin in a large, observational, non-interventional trial.

\section{METHODS}

Data from the TREAT study [44] were utilized to conduct an exploratory, post hoc analysis of the association between depression and glycemic control over 24 months in patients with T2DM initiating insulin treatment. The TREAT study was a 24-month, prospective, observational, non-interventional study that evaluated glycemic response in patients with T2DM who were initiated on insulin therapy $(N=985)$ in five European countries (Greece, Portugal, Romania, Sweden, and Turkey). Insulin-naive patients with T2DM who presented within the normal course of care and initiated insulin under the investigator's supervision or that of a referred physician were enrolled. Enrolled patients at each site had characteristics typical of patient demographics and physician specialty (primary care or specialist) for each country. Further details of the study design and clinical outcomes are available in the primary publication [44].

The TREAT study included secondary measures that are pertinent to depression and distress, including a history of diagnosis of depression, severity of depressed/anxious 
mood (EuroQol [EQ]-5D item, hereafter referred to as "depressed mood"), and diabetes-related distress (Psychological Distress domain of the Diabetes Health Profile [DHP-18]). The latter two patient-reported measures were assessed at baseline and five time points thereafter $(3,6,12$, 18, and 24 months) along with HbA1c, thus enabling a cross-sectional and longitudinal evaluation of the potential link between glycemic control, depressive mood, history of diagnosed depression, and diabetes-related distress. Using these three proxy measures of depression/distress the current study assessed: (1) at the time of insulin initiation (cross sectionally), whether depression is associated with higher HbA1c values; (2) during the 24 months following insulin initiation (longitudinally), whether depression is associated with higher HbA1c values; and (3) the relationships between the three studied proxy measures of depression/distress.

This analysis evaluated depression in three ways:

1. History of depression diagnosis-a history of depression diagnosis was recorded by the investigator at baseline ("Has the patient ever been diagnosed with any significant diagnosis other than diabetes: depression?").

2. Depressed mood-severity of depressed mood was assessed per patient self-report on the EQ-5D depression/anxiety item ("I am not anxious or depressed."; "I am moderately anxious or depressed."; "I am extremely anxious or depressed.") scored as 0 (not), 1 (moderately), or 2 (extremely). Presence of depressed mood was defined as a score of 1 or 2 .

3. Diabetes distress-diabetes-related distress was assessed by the Psychological Distress domain of the DHP-18, which included six items that asked the patients about the impact of diabetes on their mood (e.g., getting depressed, losing temper, or becoming upset, argumentative or moody), with each item being scored as 0 (never), 1 (sometimes), 2 (often), or 3 (very often).

Patient characteristics, use of oral glucoselowering medications, and diabetes disease history over the previous 12 months were recorded at baseline along with the presence of microvascular complications (diabetic retinopathy, diabetic nephropathy, diabetic neuropathy, erectile dysfunction, and amputation) and macrovascular complications (coronary heart disease, previous myocardial infract, stroke, transient ischemic attack, peripheral arterial occlusive disease, chronic heart failure, and previous coronary artery bypass graft). Insulin type at initiation was defined as long/intermediate only, mixture only, basal/bolus, or short acting only.

Two-sample $t$ tests and Fisher's exact test were used to compare baseline patient characteristics. Group comparisons on HbA1c were performed with two-sample $t$ tests at each time point (unadjusted analysis). An adjusted analysis was also performed to adjust for the effect of potential confounding variables and estimate the association that would be seen should the comparison groups (depressed vs. not depressed) be at the same level for the adjusted covariates. For depressed mood and diabetes distress, group comparisons for $\mathrm{HbA1c}$ were performed using analysis of covariance at each time point to control for the potential confounding effect of age, gender, body mass index (BMI), education, duration of diabetes, initiated insulin type, and microvascular and macrovascular complications. For comparison of HbA1c between groups with and without a history of diagnosed depression, a longitudinal, likelihood-based repeated measures mixed model analysis was used, controlling for age, 
gender, BMI, education, duration of diabetes, initiated insulin type, and microvascular and macrovascular complications. Analyses were exploratory in that they were not planned as part of the analyses supporting the original publication for this study. However, the analyses and the hypotheses they address were planned and prespecified prior to their being conducted. Results for all analyses were considered statistically significant for $p<0.05$. SAS version 9.2 (Cary, NC, USA) was used for all analyses.

\section{Compliance with Ethics}

Within the TREAT study, all treatment decisions were made between the physician and patient, and care was provided at the discretion of the physician and according to local standards of medical care. All patients provided written informed consent according to local regulations. Local requirements for ethical review and regulatory notifications, as appropriate, were met for each participating country.

The present article does not contain any new studies with human subjects performed by the authors.

\section{RESULTS}

A total of 985 patients were enrolled with a mean age of 60.39 years, mean HbA1c 9.55\%, and mean duration of 9.96 years since diagnosis of T2DM. Overall, $50.1 \%$ of patients were initiated on long/intermediate insulin, 39.3\% on mixtures, $7.8 \%$ on basal/bolus regimens, and $2.8 \%$ on short-acting insulin. Patient characteristics at baseline are summarized in Table 1. Depressed mood was reported by $49.9 \%$ of patients (43.9\% moderate, $6.1 \%$ severe) and history of depression diagnosis by $12.4 \%$. Using the cut-point of those with scores $\geq 75$ th percentile of the distribution on the DHP-18, $29.9 \%$ of patients reported high levels of diabetes-related distress at baseline.

Table 1 also shows that regardless of the depression parameter, patients with depressive symptoms were significantly more likely than patients without the depression parameter to be female, to have a higher BMI, and to have microvascular complications. In addition, patients with depressed mood had a significantly longer duration of diabetes than patients without depressed mood.

Most study participants (752; 76.3\%) have completed the 24-month study. Reasons for discontinuations $(n ; \%)$ included: lost to followup (70; 7.1\%); physician decision (70; 7.1\%); subject decision $(63 ; 6.4 \%)$; death $(22 ; 2.2 \%)$; missing (7; $0.7 \%)$; and sponsor decision (2; $0.2 \%)$. Using a comparative analysis stratified by country, the 752 study completers were not found to significantly differ from the 234 study dropouts on any of the studied baseline characteristics. There was, however, one baseline characteristic with a marginally significant difference between the completers and dropouts. There were a lower percentage of dropouts with a history of depression diagnosis at baseline compared to study completers. (9.2\% vs. $13.9 \%, p=0.054)$.

\section{Cross-Sectional Analysis at Baseline}

HbA1c was significantly (adjusted and unadjusted, $p \leq 0.001$ ) higher in patients with a history of diagnosed depression than in patients without (10.77\% vs. 9.36\%). HbA1c was similar in patients with depressed mood compared to those without depressed mood (Table 1). HbA1c was significantly higher (unadjusted, $p \leq 0.001$ ) in patients with high diabetes distress compared to patients with low distress (9.91\% vs. 9.39\%). Differences were not 


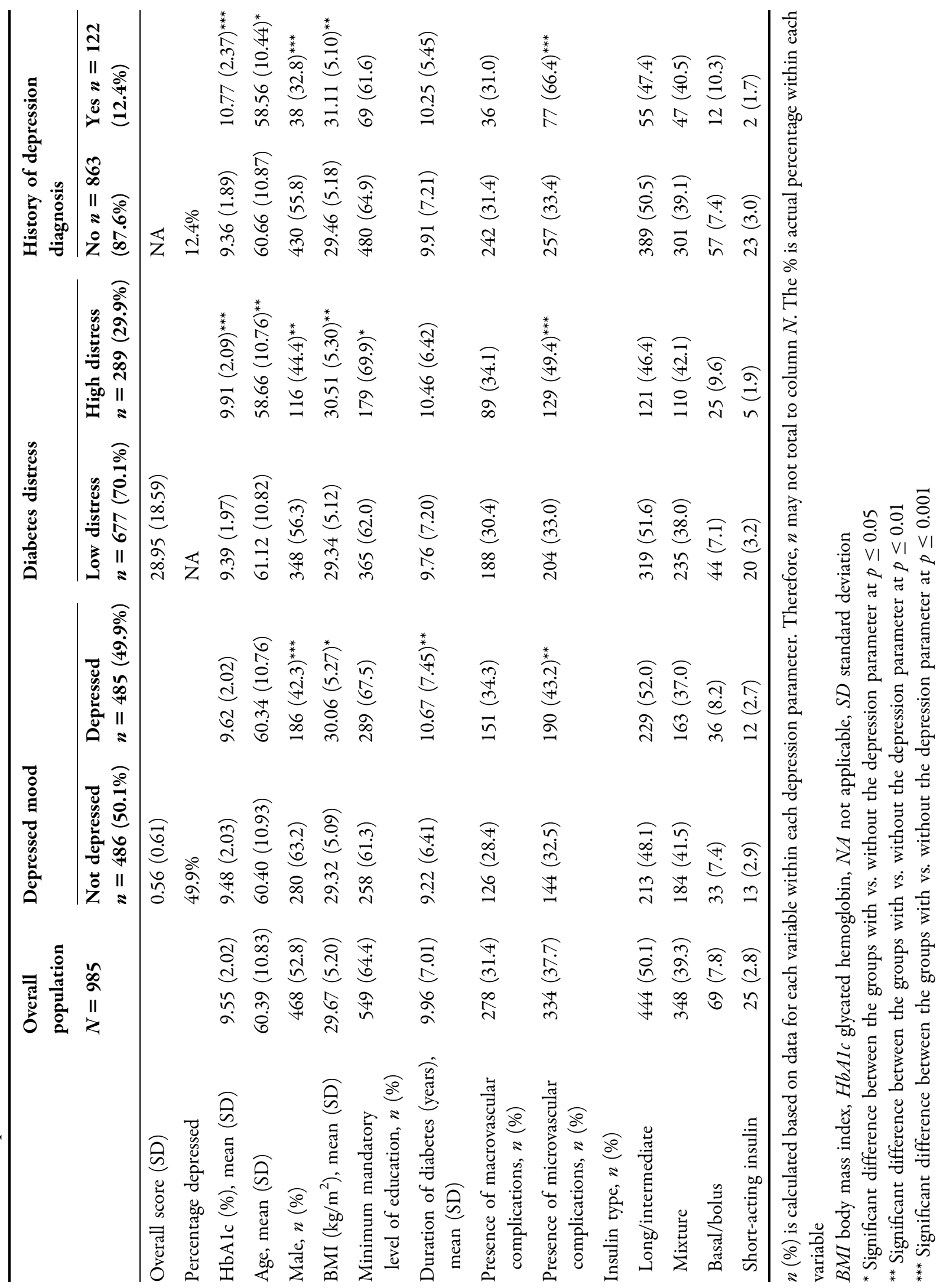


statistically significant in the adjusted analysis $(p=0.701)$.

\section{Longitudinal Analysis}

The proportion of patients with depressed mood declined over time (from $49.9 \%$ to $37.0 \%, p<0.001$; Fig. 1a), while the proportion of patients with high diabetes distress did not decline significantly over time (from $29.9 \%$ to 26.7\%, $p=0.098$; Fig. 1b).

Over the 24 months following insulin initiation, glycemic control (as measured by mean HbA1c) improved, regardless of the presence or absence of depression defined by each parameter as shown in Fig. 2. Patients with a history of diagnosed depression had significantly higher HbA1c values during the first 6 months following insulin initiation (HbA1c values decreased over time from
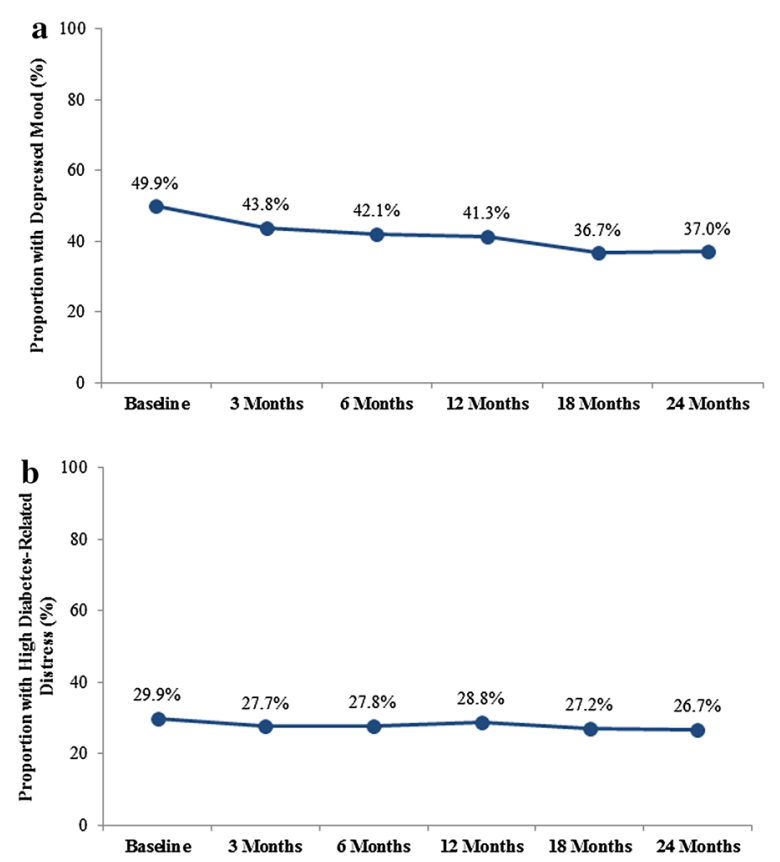

Fig. 1 a Decline over time in proportion of patients with depressed mood $[p<0.001$ for linear trend test by Generalized Estimating Equation (GEE)]. b No significant change over time ( $p=0.098$ for linear trend test by GEE) in proportion of patients with high diabetes distress
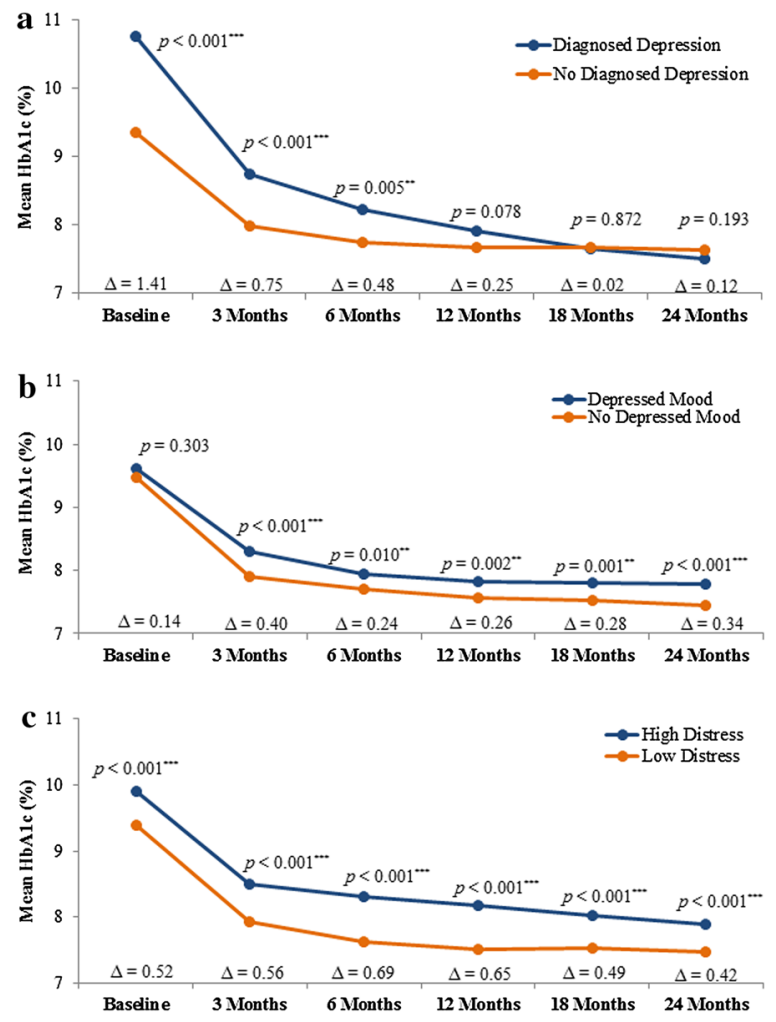

Fig. 2 a Mean HbAlc over 24 months by history of diagnosed depression at baseline. Presented results are from the unadjusted analysis. Results from the adjusted analysis showed a similar pattern as the unadjusted analysis. Group differences were, however, no longer statistically significant at 6 months and became significant in the opposite direction at 18 and 24 months. b Mean HbAlc over 24 months by depressed mood. Presented results are from the unadjusted analysis. Results from the adjusted analysis showed the same pattern as the unadjusted analysis. Group differences were, however, no longer statistically significant at 3 and 6 months. c Mean HbAlc over 24 months by diabetes distress. High diabetes distress is defined as a score 275th percentile on the DHP-18 Psychological Distress domain score at each assessment. Results are presented from the unadjusted analysis. Results from the adjusted analysis showed the same pattern as the unadjusted analysis. Group differences were, however, no longer statistically significant at baseline. DHP-18 Psychological Distress domain of the Diabetes Health Profile, HbAlc glycated hemoglobin. *Significant difference between the groups with vs. without the depression parameter at $p \leq 0.05 .{ }^{*}$ Significant difference between the groups with vs. without the depression parameter at $p \leq 0.01$. ${ }^{* * *}$ Significant difference between the groups with vs. without the depression parameter at $p \leq 0.001$. $\Delta=$ Difference between groups 
$10.77 \%$ to $8.22 \%$ ) than those without a history of diagnosis (HbA1c values decreased from 9.36\% to $7.74 \%$ ) (unadjusted $p<0.01$; Fig. $2 \mathrm{a}$ ), but not at subsequent time points (12 months, $p=0.078 ; 18$ months, $p=0.872 ; 24$ months, $p=0.193)$. Over the 24 months, patients with depressed mood continued to have higher HbA1c (HbA1c values decreased from 9.62\% to $7.79 \%$ ) compared to patients without depressed mood (HbA1c values decreased from $9.48 \%$ to 7.45\%) (unadjusted $p \leq 0.01$; Fig. $2 \mathrm{~b}$ ). Higher HbA1c values were consistently observed in patients with high diabetes distress (HbA1c values decreased from $9.91 \%$ to $7.90 \%$ ) compared to patient without high diabetes distress (HbA1c values decreased from 9.39\% to $7.48 \%$ ) throughout the study period (unadjusted $p<0.001$; Fig. 2c). Results from the adjusted analysis showed similar patterns (results not shown).

\section{Correlations Between Depression and Distress Measures}

Table 2 presents the correlations between the three depression and distress parameters over the 24 months following insulin initiation. The three measures were positively and significantly

Table 2 Correlations between the depression parameters at baseline

\begin{tabular}{ll}
\hline Depression/distress parameter & $\begin{array}{l}\text { Correlation } \\
\text { coefficient }(p \text { value })\end{array}$ \\
\hline $\begin{array}{l}\text { Depressed mood and history of } \\
\text { diagnosed depression }\end{array}$ & $0.194(p<0.001)^{\mathrm{a}}$ \\
Depressed mood and diabetes & $0.396(p<0.001)^{\mathrm{b}}$ \\
distress & \\
Diabetes distress and history of & $0.137(p<0.001)^{\mathrm{a}}$ \\
diagnosed depression & \\
\hline a Spearman correlation & \\
$\mathrm{b}$ Pearson correlation
\end{tabular}
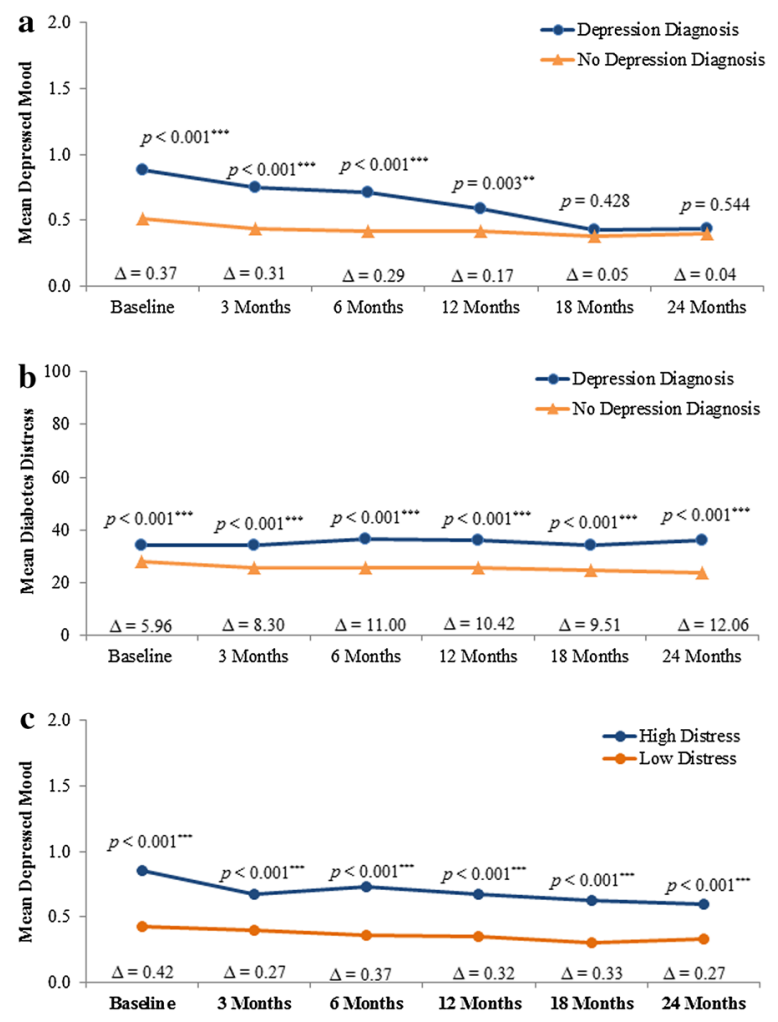

Fig. 3 a Depressed mood scores over 24 months by history of depression diagnosis at baseline. Results are presented from the unadjusted analysis. Results from the adjusted analysis showed the same pattern as the unadjusted analysis. Group differences were, however, no longer statistically significant at 12 months. b Diabetes distress scores over 24 months by history of depression diagnosis at baseline. Results are presented from the unadjusted analysis. Results from the adjusted analysis showed the same pattern as the unadjusted analysis. Group differences were, however, no longer statistically significant at baseline. c Depressed mood scores over 24 months by diabetes distress. Results are presented from the unadjusted analysis. Results from the adjusted analysis showed the same pattern as the unadjusted analysis. *Significant difference between the groups with vs. without the depression parameter at $p \leq 0.05 .{ }^{* *}$ Significant difference between the groups with vs. without the depression parameter at $p \leq 0.01$. $^{* * *}$ Significant difference between the groups with vs. without the depression parameter at $p \leq 0.001 . \Delta=$ Difference between groups

correlated at baseline, although the magnitude of the associations was relatively low, with the higher correlation observed between depressed mood and diabetes distress $(r=0.396)$. Figure 3 
shows the longitudinal relationships between the depression parameters. In the first 12 months post-initiation of insulin therapy, patients with a history of diagnosed depression had significantly higher mean scores on the depressive mood item compared to patients without a history of diagnosed depression (Fig. 3a). Similarly, Fig. 3b and $\mathrm{c}$ shows relationships between diabetes distress and history of diagnosed depression, and depressed mood by baseline diabetesrelated distress. In all cases, the presence of depression at baseline is associated with the presence of more symptoms over time. Results from the adjusted analysis showed essentially the same pattern (results not shown).

\section{Sensitivity Analyses}

As study participants were enrolled from five European countries (Greece, Portugal, Romania, Sweden, and Turkey), we also assessed-as a sensitivity analysis-the potential impact of country on the current results by repeating the original analyses in two ways: we first reanalyzed the findings while stratifying by country and then repeated the original analysis while adding country to the covariates in the model. Results (not shown) were essentially unchanged when stratifying by country and when adding country to the covariate list. There were, however, four changes from the original $p$ values (out of 36 new comparisons): two previously significant comparisons became non-significant and two previously non-significant comparisons became significant.

\section{DISCUSSION}

This exploratory, post hoc analysis found consistent and significant associations between poorer glycemic control and each of the three depression and distress parameters: history of depression diagnosis, depressed mood, and diabetes distress in T2DM patients who initiated insulin therapy. This analysis also demonstrated that patients with higher depression parameters or distress at baseline had significantly higher rates of microvascular complications. Poor glycemic control has indeed been associated with development of diabetic complications [45], and these complications are probably a more direct cause of depressive mood in patients with higher HbA1c than HbA1c alone. In addition, these results show that initiation of insulin therapy was associated with improved glycemic control, and the proportion of patients with preexisting depressed mood decreased significantly (by 13\% overall from $49.9 \%$ to $37.0 \%$ ) and resulted in numerically, but not statistically significant, reductions in the proportion of patients with preexisting high diabetes distress (from 29.9\% to $26.7 \%, p=0.098$ ).

Consistent with prior research, a substantial proportion of patients with T2DM in this cohort were experiencing depression or emotional distress, as measured by the three proxy measures of depression. History of depression diagnosis was present in $12.4 \%$ of the patients, a finding congruent with previous studies that estimated the prevalence of major depression in diabetes to be around $12 \%$ (ranging from $8 \%$ to 18\%) $[2,46,47]$. Depressed mood was reported by $50 \%$ of patients and diabetes-related distress by $30 \%$.

Although the positive associations between poorer glycemic control and depression parameters were observed at baseline for only two of the three depression parameters (history of depression diagnosis and diabetes distress), there was a higher rate of microvascular complications for all three parameters. While the mean HbA1c values improved for patients 
during the 24 months following insulin initiation, glycemic control was poorer for patients with, compared to without, the depressive parameters. A higher HbA1c at baseline is, however, associated with a higher HbA1c at endpoint after insulin treatment, although the decrease in HbA1c is higher [48, 49]. A higher HbA1c at baseline was also associated with higher $\mathrm{HbA1c}$ at endpoint in the TREAT study [44]. In this analysis, insulin regimens were similar between groups with or without depressive parameters, and thus a higher HbA1c at endpoint is not unexpected and probably more linked to the difficulty and need of more complex insulin regimens in patients with worse glycemic control at insulin initiation than to depressive parameters.

The consistent findings may be due, at least in part, to the positive and significant interrelationships between the depression parameters. Although the correlations were relatively small in magnitude, the correlation between diabetes distress and depressed mood was higher $(r=0.396)$. This is consistent with prior research [28], which reported a correlation of $r=0.48$ between depressed mood and diabetes distress. Therefore, current findings suggest that the different depression parameters may capture a different but interrelated condition that may require additional assessment and management to optimize glycemic control in patients with T2DM.

In addition, the importance of identifying a history of depression diagnosis has been highlighted by the finding that patients with a history of depression diagnosis had higher levels of depressed mood and diabetes distress not only at baseline, but also during the followup period. Interestingly, this differentiation persisted over the 24-month study for diabetes distress but did not persist for depressed mood beyond the 12-month assessment. Patients with or without a history of depression diagnosis no longer differed on their level of depressed mood after 12 months of insulin therapy, suggesting that those with a preexisting depression diagnosis may have achieved remission, as their levels of depressive symptoms did not differ from patients without a history of depression diagnosis.

This exploratory, post hoc analysis has a number of potential limitations. First is the post hoc nature of the analysis, as the TREAT study was originally designed to assess glycemic response in T2DM patients initiating insulin. Another limitation is the proxy nature of the studied depression parameters. None of the measures used in the analysis were developed or validated for use as a standalone measure of depression, thus the psychometric properties of these depression parameters for this use are unknown. A third limitation relates to the history of depression diagnosis at baseline, which does not offer information regarding the specific type of depression diagnosis or when the diagnosis was made. Moreover, no information was available on patients' use of antidepressants at baseline during the study follow-up period, and the use of antidepressants would be expected to impact patients' level of depression and anxiety. Furthermore, high versus low diabetes distress was distinguished by a threshold score of $\geq 75 \%$ in the psychological distress score distribution. We chose this threshold based on the statistical distribution and not on any concurrent validation with other measures of distress.

An additional limitation of the study is that we conducted analyses using observed data. It is not possible to rule out that the missing data 
(through dropouts and missing visits) show informative missingness and that the results may be different if we had complete data on every subject that entered the study.

The study strengths include the longitudinal measurement of $\mathrm{HbA1c}$ and multiple affective measures available at six time points over a 24-month period, which enabled the assessment of both cross-sectional and longitudinal relationships between glycemic control and affective parameters. Reported results reflected adjusted as well as unadjusted analyses, which helped address a number of core baseline differences between those with and without the affective measure. Another strength is the focus on patients initiating insulin.

\section{CONCLUSIONS}

Current findings of a decline in the proportion of patients with depressive mood from preinitiation to post-initiation of insulin therapy may suggest that once the barriers associated with insulin treatment have been addressed, depressed mood improves with better glycemic control. A better understanding of the relationship between glycemic control, diabetic complications, and specific affective parameters may help clinicians to focus more attention on screening for affective conditions and provide information that may lead to more timely treatment or referrals to both mental health and diabetes professionals. In addition to the potential beneficial impact on clinical practice and patients' outcomes, such information may also be useful for diabetes research, as it may help highlight the need to evaluate specific potential affective confounders when assessing glycemic control among patients with T2DM, in either cross-sectional or longitudinal manner.

\section{ACKNOWLEDGMENTS}

This study and article processing charges were funded by Eli Lilly and Company, Indianapolis, IN, USA. Writing assistance was provided by Dr. Jarrett Coffindaffer and Ms. Rebecca McCracken of inVentiv Health Clinical, and editorial assistance was provided by Ms. Teri Tucker of inVentiv Health Clinical. Support for this assistance funded by Eli Lilly and Company. All named authors meet the International Committee of Medical Journal Editors (ICMJE) criteria for authorship for this manuscript, take responsibility for the integrity of the work as a whole, and have given final approval to the version to be published.

Conflict of interest. Haya Ascher-Svanum is an employee and stockholder of Eli Lilly and Company. Anthony Zagar is an employee and stockholder of Eli Lilly and Company. Dingfeng Jiang is an employee and stockholder of Eli Lilly and Company. Dara Schuster is an employee and stockholder of Eli Lilly and Company. Henry Schmitt is an employee and stockholder of Eli Lilly and Company. Ellen B. Dennehy is an employee and stockholder of Eli Lilly and Company. David M. Kendall is an employee and stockholder of Eli Lilly and Company. Joel Raskin is an employee and stockholder of Eli Lilly and Company. Robert J. Heine is an employee and stockholder of Eli Lilly and Company.

Compliance with ethics guidelines. This article does not contain any new studies with human subjects performed by the authors.

Open Access. This article is distributed under the terms of the Creative Commons Attribution Noncommercial License which permits any noncommercial use, distribution, and reproduction in any medium, provided the original author(s) and the source are credited. 


\section{REFERENCES}

1. Bot M, Pouwer F, Zuidersma M, van Melle JP, de Jonge $\mathrm{P}$. Association of coexisting diabetes and depression with mortality after myocardial infarction. Diabetes Care. 2012;35:503-9.

2. Andreoulakis E, Hyphantis T, Kandylis D, Iacovides A. Depression in diabetes mellitus: a comprehensive review. Hippokratia. 2012;16:205-14.

3. Lustman PJ, Anderson RJ, Freedland KE, de Groot M, Carney RM, Clouse RE. Depression and poor glycemic control: a meta-analytic review of the literature. Diabetes Care. 2000;23:934-42.

4. de Groot M, Anderson R, Freedman KE, Clouse RE, Lustman PJ. Association of depression and diabetes complications: a meta-analysis. Psychosom Med. 2001;63:619-30.

5. Egede LE, Nietert PJ, Zheng D. Depression and allcause and coronary heart disease mortality among adults with and without diabetes. Diabetes Care. 2005;28:1339-45.

6. Ismail K, Winkley K, Stahl D, Chalder T, Edmonds M. A cohort study of people with diabetes and their first foot ulcer: the role of depression on mortality. Diabetes Care. 2007;30:1473-9.

7. Schmitz N, Gariépy G, Smith KJ, et al. Recurrent subthreshold depression in type 2 diabetes: an important risk factor for poor health outcomes. Diabetes Care. 2014;37:970-8.

8. Fisher L, Mullan JT, Arean P, Glasgow RE, Hessler D, Masharani U. Diabetes distress but not clinical depression or depressive symptoms is associated with glycemic control in both cross-sectional and longitudinal analyses. Diabetes Care. 2010;33:23-8.

9. Lustman PJ, Clouse RE. Depression in diabetes: the chicken or the egg? Psychosom Med. 2007;69:297-9.

10. McKellar JD, Humphreys K, Plette JD. Depression increases diabetes symptoms by complicating patients' self-care adherence. Diabetes Educ. 2004;30:485-92.

11. Katon WJ, Von Korff MV, Lin EH, et al. The Pathways Study: a randomized trial of collaborative care in patients with diabetes and depression. Arch Gen Psychiatry. 2004;61:1042-9.

12. Trief PM, Morin PC, Izquierdo R, et al. Depression and glycemic control in elderly ethnically diverse patients with diabetes: the IDEATel project. Diabetes Care. 2006;29:830-5.
13. Pouwer F, Geelhoed-Duijvestijn PH, Tack CJ, et al. Prevalence of comorbid depression is high in outpatients with Type 1 or Type 2 diabetes mellitus. Results from three out-patient clinics in the Netherlands. Diabet Med. 2010;27:217-24.

14. Van Tilburg MA, McCaskill CC, Lane JD, et al. Depressed mood is a factor in glycemic control in type 1 diabetes. Psychosom Med. 2001;63:551-5.

15. Ciechanowski PS, Katon WJ, Russo JE, Hirsch IB. The relationship of depressive symptoms to symptom reporting, self-care and glucose control in diabetes. Gen Hosp Psychiatry. 2003; 25:246-52.

16. Surwit RS, van Tilburg MA, Parekh PI, Lane JD, Feinglos MN. Treatment regimen determines the relationship between depression and glycemic control. Diabetes Res Clin Pract. 2005;69:78-80.

17. Paschalides C, Wearden AJ, Dunkerley R, Bundy C, Davies R, Dickens CM. The associations of anxiety, depression and personal illness representations with glycaemic control and health-related quality of life in patients with type 2 diabetes mellitus. J Psychosom Res. 2004;57:557-64.

18. Egede LE, Ellis C, Grubaugh AL. The effect of depression on self-care behaviors and quality of care in a national sample of adults with diabetes. Gen Hosp Psychiatry. 2009;31:422-7.

19. Katon W, Russo J, Lin EH, et al. Depression and diabetes: factors associated with major depression at five-year follow-up. Psychosomatics. 2009;50:570-9.

20. Papelbaum M, Moreira RO, Coutinho $\mathrm{W}$, et al. Depression, glycemic control and type 2 diabetes. Diabetol Metab Syndr. 2011;3:26-9.

21. Wagner JA, Abbott GL, Heapy A, Yong L. Depressive symptoms and diabetes control in African Americans. J Immigr Minor Health. 2009;11:66-70.

22. Richardson LK, Egede LE, Mueller M, Echols CL, Gebregziabher M. Longitudinal effects of depression on glycemic control in veterans with Type 2 diabetes. Gen Hosp Psychiatry. 2008;30:509-14.

23. Dirmaier J, Watzke B, Koch U, et al. Diabetes in primary care: prospective associations between depression, nonadherence and glycemic control. Psychother Psychosom. 2010;79:172-8.

24. Aikens JE, Perkins DW, Lipton B, Piette JD. Longitudinal analysis of depressive symptoms and glycemic control in type 2 diabetes. Diabetes Care. 2009;32:1177-81. 
25. Heckbert SR, Rutter CM, Oliver M, et al. Depression in relation to long-term control of glycemia, blood pressure, and lipids in patients with diabetes. J Gen Intern Med. 2010;25:524-9.

26. Kovacs BK, Nicolucci A, Holt RI, DAWN2 Study Group, et al. Diabetes Attitudes, Wishes and Needs second study (DAWN2 $2^{\mathrm{TM}}$ ): cross-national benchmarking indicators for family members living with people with diabetes. Diabet Med. 2013;30:778-88.

27. Fisher L, Skaff MM, Mullan JT, et al. Clinical depression versus distress among patients with type 2 diabetes: not just a question of semantics. Diabetes Care. 2007;30:542-8.

28. Fisher L, Glasgow RE, Strycker LA. The relationship between diabetes distress and clinical depression with glycemic control among patients with type 2 diabetes. Diabetes Care. 2010;33:1034-6.

29. Fisher L, Hessler DM, Polonsky WH, Mullan J. When is diabetes distress clinically meaningful? Establishing cut points for the Diabetes Distress Scale. Diabetes Care. 2012;35:259-64.

30. Nicolucci A, Kovacs BK, Holt RI, et al. DAWN2 Study Group. Diabetes Attitudes, Wishes and Needs second study (DAWN2 $\left.{ }^{\mathrm{TM}}\right)$ : cross-national benchmarking of diabetes-related psychosocial outcomes for people with diabetes. Diabet Med. 2013;30:767-77.

31. Fisher L, Hessler D, Glasgow RE, et al. REDEEM: a pragmatic trial to reduce diabetes distress. Diabetes Care. 2013;36:2551-8.

32. Peyrot $M$, Rubin $R R$, Lauritzen $T$, International DAWN Advisory Panel, et al. Resistance to insulin therapy among patients and providers: results of the cross-national Diabetes Attitudes, Wishes, and Needs (DAWN) study. Diabetes Care. 2005;28:2673-9.

33. Polonsky WH, Fisher L, Guzman S, Villa-Caballero L, Edelman SV. Psychological insulin resistance in patients with type 2 diabetes: the scope of the problem. Diabetes Care. 2005;28:2543-5.

34. Dailey G. Optimum management of type 2 diabetes-timely introduction, optimization and intensification of basal insulin. Diabetes Obes Metab. 2008;10(Suppl 2):5-13.

35. Khunti K, Wolden ML, Thorsted BL, Andersen M, Davies MJ. Clinical inertia in people with type 2 diabetes: a retrospective cohort study of more than 80,000 people. Diabetes Care. 2013;36:3411-7.

36. Casagrande SS, Fradkin JE, Saydah SH, Rust KF, Cowie CC. The prevalence of meeting A1C, blood pressure, and LDL goals among people with diabetes, 1988-2010. Diabetes Care. 2013;36:2271-9.

37. Bloomgarden ZT. Achieving glycemic goals in type 2 diabetes. Diabetes Care. 2007;30:174-80.

38. Khan H, Lasker SS, Chowdhury TA. Prevalence and reasons for insulin refusal in Bangladeshi patients with poorly conrolled Type 2 diabetes in East London. Diabet Med. 2008;25:1108-11.

39. Larkin ME, Capasso VA, Chen CL, et al. Measuring psychological insulin resistance: barriers to insulin use. Diabetes Educ. 2008;34:511-7.

40. Karter AJ, Subramanian U, Saha C, et al. Barriers to insulin initiation: the translating research into action for diabetes insulin starts project. Diabetes Care. 2010;33:733-5.

41. Sharma SK, Yeo JP, Garber A. Lessons in initiating insulin in clinical practice. Diabetes Res Clin Pract. 2008;81(Suppl 1):S16-22.

42. Hermanns N, Kulzer B, Krichbaum M, Kubiak T, Haak T. How to screen for depression and emotional problems in patients with diabetes: comparison of screening characteristics of depression questionnaires, measurement of diabetes-specific emotional problems and standard clinical assessment. Diabetologia. 2006;49:469-77.

43. Holt RI, de Groot M, Lucki I, Hunter CM, Sartorius $\mathrm{N}$, Golden SH. NIDDK international conference report on diabetes and depression: current understanding and future directions. Diabetes Care. 2014;37:2067-77.

44. Oguz A, Benroubi M, Brismar K, et al. Clinical outcomes after 24 months of insulin therapy in patients with type 2 diabetes in five countries: results from the TREAT study. Curr Med Res Opin. 2013;29:911-20.

45. Mezuk B, Eaton WW, Albrecht S, Golden SH. Depression and type 2 diabetes over the lifespan: a meta-analysis. Diabetes Care. 2008;31:2383-90.

46. Stratton IM, Adler AI, Neil HA, et al. Association of glycaemia with macrovascular and microvascular complications of type 2 diabetes (UKPDS 35): prospective observational study. BMJ. 2000;321:405-12.

47. Nouwen A, Winkley K, Twisk J, et al. Type 2 diabetes mellitus as a risk factor for the onset of depression: a systematic review and meta-analysis. Diabetologia. 2010;53:2480-6.

48. Esposito K, Chiodini P, Bellastella G, Maiorino MI, Giugliano D. Proportion of patients at HbA1c target 
$<7 \%$ with eight classes of antidiabetic drugs in type 2 diabetes: systematic review of 218 randomized controlled trials with 78945 patients. Diabetes Obes Metab. 2012;14:228-33.

49. Riddle MC, Vlajnic A, Zhou R, Rosenstock J. Baseline HbA1c predicts attainment of $7.0 \%$
HbA1c target with structured titration of insulin glargine in type 2 diabetes: a patient-level analysis of 12 studies. Diabetes Obes Metab. 2013;15:819-25. 